OPEN ACCESS

Edited by:

Adam Hirsh,

Indiana University, Purdue University Indianapolis, United States

Reviewed by:

Peter Drummond,

Murdoch University, Australia Bernadette Ann Murphy,

Ontario Tech University, Canada

${ }^{*}$ Correspondence:

Catherine Mercier

catherine.mercier@rea.ulaval.ca

Specialty section:

This article was submitted to Virtual Reality in Medicine, a section of the journal Frontiers in Virtual Reality

Received: 12 April 2021 Accepted: 16 June 2021 Published: 29 June 2021

Citation: Brun C, Pinard AM, McCabe CS and Mercier C (2021) Virtual RealityInduced Sensorimotor Conflict Evokes Limb-Specific Sensory Disturbances in Complex Regional Pain Syndrome.

Front. Virtual Real. 2:694293. doi: 10.3389/frvir.2021.694293

\section{Virtual Reality-Induced Sensorimotor Conflict Evokes Limb-Specific Sensory Disturbances in Complex Regional Pain Syndrome}

\author{
Clémentine Brun ${ }^{1,2}$, Anne Marie Pinard ${ }^{3,4}$, Candida S. McCabe ${ }^{5,6,7}$ and Catherine Mercier ${ }^{1,2 *}$ \\ ${ }^{1}$ Center for Interdisciplinary Research in Rehabilitation and Social Integration (CIRRIS), Quebec City, QC, Canada, ${ }^{2}$ Department of \\ Rehabilitation, Laval University, Quebec City, QC, Canada, ${ }^{3}$ Department of Anesthesiology, Laval University, Quebec City, QC, \\ Canada, ${ }^{4}$ Department of Anesthesiology and Intensive Care, CHU de Québec-Université Laval, Quebec City, QC, Canada, \\ ${ }^{5}$ Dorothy House Hospice, Winsley, United Kingdom, ${ }^{6} \mathrm{HAS}$ - Nursing and Midwifery, University of the West of England, Bristol, \\ United Kingdom, ${ }^{7}$ Florence Nightingale Foundation, London, United Kingdom
}

The origin of sensory disturbances in complex regional pain syndrome (CRPS) remains unclear. It has been hypothesized that such disturbances are due to attentional effects and/or sensorimotor integration deficits. If sensory disturbances are explained by sensorimotor integration deficits, they would be expected to be specific in terms of the category of sensation evoked and in terms of localization. Objective 1: To test whether sensory disturbances evoked by a unilateral sensorimotor conflict are specific to the painful limb and differ according to the category of sensory disturbances in individuals with a unilateral CRPS compared to healthy controls $(\mathrm{HC})$. Objective 2: To assess the association between clinical characteristics and conflict-induced sensory disturbances. Objective 3: To assess conflict-induced motor disturbances. Ten adults with upper limb (UL) CRPS and $23 \mathrm{HC}$ were recruited. Sensorimotor conflict was elicited with a KINARM exoskeleton interfaced with a 2D virtual environment allowing the projection of a virtual UL that was moving in either a congruent or incongruent manner relative to the actual UL movement. Participants rated sensory disturbances from 0 (no change) to 3 (high change) on a 8-item questionnaire. Items were classified into two Categories (Category 1: pain, discomfort, the feeling of losing a limb, change in weight and temperature; Category 2: feelings of peculiarity, the impression of gaining a limb and losing control). Motor disturbances were quantified as mediolateral drift and changes in amplitude of UL movement. Clinical characteristics included the intensity and duration of pain, proprioception, and body perception. CRPS participants report higher Category 1 than Category 2 disturbances for the Affected limb (while the reverse was observed for $\mathrm{HC}$ and for the Unaffected limb). In addition, no difference was observed between the Unaffected limb in CRPS and the Dominant limb in HC for Category 2 disturbances, while higher conflict sensitivity was observed for Category 1 disturbances. Conflict sensitivity was only related to higher pain for Category 1 disturbances in the Affected limb. Finally, no effect on motor

Abbreviations: CRPS, complex regional pain syndrome; UL, upper limb; HC, healthy controls; VF, visual feedback. 
disturbances was observed. While they do not completely rule out the attentional hypothesis, these results support the hypothesis of sensorimotor integration deficits.

Keywords: sensory disturbances, CRPS, chronic pain, virtual reality, robotics, sensorimotor integration

\section{INTRODUCTION}

Complex regional pain syndrome (CRPS) is a chronic pain condition characterized by disproportionate pain to the original injury accompanied by sensory, motor and autonomic dysfunctions (Harden et al., 2007). In this population, sensory disturbances are frequently observed and are characterized by changes in the perception of limb weight and temperature, a distorted mental image of the affected limb, feeling the limb as a foreign body part with a pronounced disliking and a desire to amputate it (Förderreuther et al., 2004; Lewis et al., 2007). It is important to note that sensory disturbances are also observed in other chronic pain conditions, but are clearly more pronounced in CRPS (Förderreuther et al., 2004; Frettlöh et al., 2006), and occur mainly in the affected limb (Lewis et al., 2007). In parallel, several studies demonstrates that sensory deficits (changes in sensation, i.e. the stimulus detection by the sensory system) measured with quantitative sensory testing (as hyperalgesia, hyperesthesia, etc.) extend beyond the affected limb (Rommel et al., 2001; Drummond et al., 2018; Palmer et al., 2019), but this has not been investigated for sensory disturbances (changes in perception, i.e. the interpretation/the meaning of the sensory stimuli). Moreover, the reasons why people with CRPS report such sensory disturbances still remain unclear.

It has been shown that sensory disturbances are positively associated with anxiety and pain-catastrophizing (Michal et al., 2017), and some authors suggested that CRPS represents an excessive response to perceived threat of tissue injury (Bean et al., 2015). Considering the fact that a perceived threat leads to attentional bias in anxiety disorders (Cisler and Koster, 2010), it has been suggested that sensory disturbances are due to attentional effects (Förderreuther et al., 2004; Frettlöh et al., 2006; Michal et al., 2017).

Other theories suggest that CRPS sensory disturbances are explained by cortical reorganizations (McCabe and Blake, 2008) that are observed in the primary somatosensory (Di Pietro et al., 2013) and motor (Maihöfner et al., 2003) cortices, and in the posterior parietal cortex (Vartiainen et al., 2009). These cortical reorganizations might affect sensorimotor integration since the motor command and the related sensory feedback are closely coupled to monitor movements. It has been hypothesized that sensory disturbances, including painful sensations, are due to an incongruence between the predicted and the actual sensory feedback, named as sensorimotor conflict (Harris, 1999; McCabe et al., 2009). To demonstrate that point, numerous studies experimentally created sensorimotor conflicts. Such conflicts have been shown to induce sensory disturbances in various pain populations (Don et al., 2016), including in CRPS (Brun et al., 2018c). These sensory disturbances are qualitatively similar to the sensory disturbances that are spontaneously (i.e., in the absence of experimental manipulation) reported by the patients (McCabe et al., 2007).

If sensory disturbances are explained by cortical reorganization and sensorimotor integration deficits rather than by attentional factors, they would be expected to be specific in terms of the category of sensation evoked and in terms of localization. Attentional factors such as arousal or hypervigilance could result in more general and diffuse effects, for example effects relative to other sensory domains (visual, hearing, smelling, etc.) and beyond the affected body part (McDermid et al., 1996). In CRPS, higher discomfort also extends to auditory stimuli (Knudsen et al., 2011). Recent studies identified two distinct categories of sensory disturbances evoked by sensorimotor conflict (Category 1: pain, discomfort, lost limb, temperature, weight; Category 2: peculiarity, losing control, extra-limb) that are differentially affected by the presence of pain (Brun et al., 2017; Brun et al., 2018c). Indeed, conflict-induced Category 1 disturbances are more intense in people with CRPS and fibromyalgia syndrome compared to pain-free individuals, while conflict-induced Category 2 disturbances do not differ between groups. This specificity, in the pattern of sensations evoked, suggests that sensory disturbances arise from sensorimotor processes rather than from attentional effects. Another way of addressing the specificity of the sensory disturbances evoked by conflicts would be to compare sensory disturbances evoked in a painful vs. nonpainful site. This has not been done so far as previous studies were either conducted using a mirror (involving both upper or lower limbs) (Don et al., 2016) or using virtual reality (which provide much more flexible ways of inducing a conflict during unilateral movement) but in a population with generalized pain (fibromyalgia) (Brun et al., 2020).

Therefore, the first objective of the study was to assess whether sensory disturbances evoked by a unilateral sensorimotor conflict are specific to the painful limb and differ according to the Category of sensory disturbances in individuals with a unilateral CRPS compared to healthy controls (HC). We hypothesized that CRPS participants would report higher Category 1 disturbances than Category 2 for the Affected limb, and that a reverse pattern (Category 2 disturbances $>$ Category 1 disturbances) would be observed for the Unaffected limb and HC, based on results of previous studies (Brun et al., 2017; Brun et al., 2018c).

A second objective was to assess the association between clinical characteristics and sensory disturbances evoked by a unilateral sensorimotor conflict. Clinical characteristics considered included the intensity and duration of pain, proprioception deficits, and body perception disturbances.

A third objective was to assess motor disturbances induced by a unilateral sensorimotor conflict as several studies showed increased motor disturbances during sensorimotor conflicts 
A

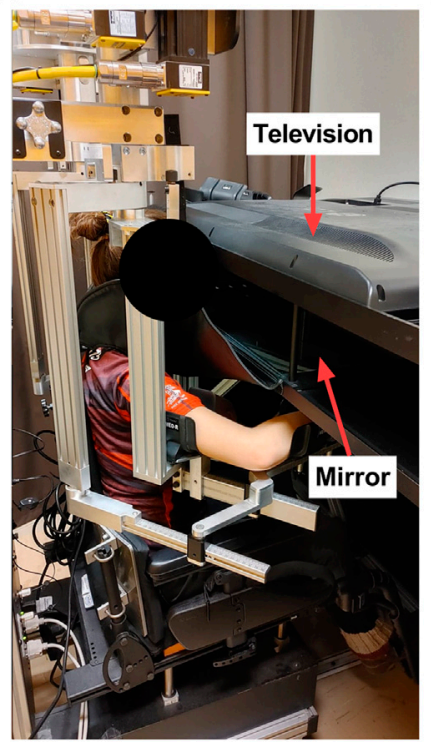

B
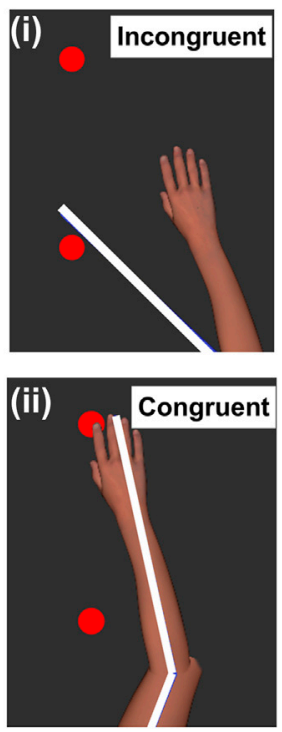

FIGURE 1 | Experimental set up. (A): The KINARM is a robotized exoskeleton allowing upper limbs movement in the horizontal plane. A 2D virtual environment is interfaced with the robot allowing to project a virtual upper limb that is moving either in a congruent or incongruent manner relative to the actual upper limb movement of the participant. In order to have an appropriate vision of depth of the virtual upper limb, a mirror is positioned at halfway between the actual upper limb and the television. (B): The virtual upper limb moving either in incongruent (i) or incongruent manner (ii). The white lines depict the actual position of the upper limb.

(Brun et al., 2017; Brun et al., 2020) and motor impairments in CRPS (Maihöfner et al., 2003; Bank et al., 2013). We hypothesized that motor disturbances induced by the conflict would be higher for the Affected limb compared to the Unaffected limb and HC.

\section{MATERIALS AND METHODS}

\section{Participants and Ethics Statement}

Ten adults with a unilateral upper limb CRPS [diagnosed according to the Budapest criteria (Harden et al., 2010)] and $23 \mathrm{HC}$ matched in age and sex volunteered. CRPS participants were excluded if they had motor impairments interfering with the experimental task $\left(80^{\circ}\right.$ of shoulder abduction and forward movements with an amplitude of $30 \mathrm{~cm}$, with the weight of the arm being fully supported). Control participants were excluded if they had chronic pain in the last three months or acute UL pain on the day of experimentation. Participants with uncorrected visual impairments were excluded (for both groups).

CRPS participants were recruited from the outpatient clinic at the Center of Expertise in Chronic Pain Management (CHU de Québec-Université Laval, Quebec City, QC, Canada). Controls were recruited from Laval University.

All participants provided written informed consent before enrollment. This study was approved by the local ethical review board (Centre intégré universitaire en santé et services sociaux de la Capitale Nationale, no. 2015-461) and conformed with the Declaration of Helsinki.

For the CRPS group, a brief history of each patient's condition (time since diagnosis, pain manifestations, pharmacological and non-pharmacological treatments, comorbidities) was obtained from a semi-structured interview at the beginning of the experimental session.

\section{Experimental Design}

All participants took part in one single session lasting approximately $1 \mathrm{~h}$. Sensory and motor disturbances induced by sensorimotor conflict were assessed in the same experimental task. Participants were exposed to two experimental conditions (see Procedure for more details): Congruent or Incongruent visual feedback (VF). ULs were tested in a counterbalanced order across each group. Before the experimental task, all participants completed a proprioceptive task, and answered questions about pain intensity and body perception disturbances (see Clinical Characteristics).

\section{Material}

Sensorimotor conflict was elicited with a KINARM robotized exoskeleton [KINARM, Kingston ON, Canada, see (Brun et al., 2020)] that allows UL movements in the transversal plane by means of elbow flexion-extension and shoulder abductionadduction (see Figure 1). A 2D virtual environment (47 inch) was interfaced with the robot allowing the projection of a virtual UL (with appropriate vision of depth) that was moving in either a congruent or incongruent manner relative to the actual UL movement (Dexterit-E software version 3.5.3). Importantly, participant's ULs were obstructed from view in all experimental conditions. Joint angular positions for shoulder and elbow were obtained from the KINARM motor encoders and sampled at $1 \mathrm{kHz}$. The position of the index was computed in real time. Data processing was made with Matlab (MathWorks, R2011b). The KINARM was also used to assess proprioception using a KINARM Arm position matching task (Dukelow et al., 2010), a standard test that has previously been used to assess proprioception in various clinical conditions (Kuczynski et al., 2014; Brun et al., 2020), including CRPS (Brun et al., 2018b).

\section{Experimental Conditions and Procedure}

The experimental task was similar to previous studies in our lab (Brun et al., 2018a, 2020).

Participants were exposed to two experimental conditions in which participants were not allowed to look away from the $2 \mathrm{D}$ environment or to close their eyes. During the Congruent VF condition, the virtual UL reproduced faithfully the participant's actual movement (Figure 1). During the Incongruent VF condition, the virtual UL followed a pre-defined trajectory in the mediolateral axis with a $30 \mathrm{~cm}$ amplitude, while the participant performed a movement in the transversal axis with the same amplitude.

Each condition was repeated twice per UL in a pseudorandom order and followed the same time course. Firstly, two targets $(2 \mathrm{~cm}$ diameter $)$ were projected on the virtual 
environment and were positioned $30 \mathrm{~cm}$ away from each other, being equidistant from the initial position of the participant UL, which corresponded to a fixed angular position of the elbow $\left(90^{\circ}\right)$ and shoulder $\left(30^{\circ}\right)$. Secondly, a metronome beat indicated the beginning of the movement and participants were required to reach each target successively, without stopping on them, in order to create a cyclic movement as fluid and as straight as possible. The frequency of the metronome beat was $0.33 \mathrm{~Hz}$, allowing to control UL movement velocity. Each trial was divided into two phases. In the Baseline phase (21 s), congruent feedback was provided. In the Experimental phase (21 s), participants were exposed to Congruent or Incongruent VF.

\section{Measures and Data Analysis}

After each trial, participants had to verbally respond to a questionnaire about their perception of their UL using an eight-item questionnaire (Brun et al., 2018a). Questions were about pain, discomfort, the perception of losing a limb, having an extra limb, change in weight and/or temperature, feelings of peculiarity and losing control. Each question was rated from 0 to 3 as following: $0=$ no change of perception from the Baseline phase to the Experimental phase, $1=$ low change, $2=$ medium change, 3 = high change. Sensory disturbances were divided into two distinct subgroups (Brun et al., 2017; 2018c): Category 1 = pain, discomfort, lost limb, temperature, weight; Category 2 = peculiarity, losing control, extra limb.

Each Category of sensory disturbances was expressed as a change between the Congruent and the Incongruent VF, termed as Conflict sensitivity. A positive value indicates higher sensory disturbances during Incongruent VF compared to Congruent VF.

Motor disturbances were assessed using two variables based on the position of the index fingertip (computed by the KINARM): Amplitude and mediolateral Drift (Brun et al., 2018a). For the Amplitude, anteroposterior coordinates were computed for each peak of flexion and extension. For each movement half-cycle, the amplitude on the y-axis was extracted. For the Drift, for each movement half-cycle, the mediolateral coordinates of the maximal deviant point (from the virtual straight line between both targets) were extracted. Both motor outcomes were expressed as a change from the Baseline phase to the Experimental phase. A positive value indicates higher Drift/ Amplitude in the Experimental phase compared to the Baseline phase and vice versa for a negative value.

\section{Clinical Characteristics}

Pain intensity: Two ratings of pain were obtained using a numerical pain rating scale from 0 (no pain) to 10 (worst pain), reflecting pain intensity: over the last $24 \mathrm{~h}$ and over the last week.

Pain duration: Pain duration was measured in months since the onset of pain.

Proprioception: Proprioception was assessed with the KINARM for the left and the right UL in a counterbalanced order. In this task, no VF was provided, and participants had to match the position of the right UL with the left UL (and vice versa for the assessment of the left UL). To do that, the tested UL was passively moved by the robot to one of the four pre-defined positions relative to the angular positions of the shoulder $\left(30^{\circ}\right)$ and elbow $\left(90^{\circ}\right)$ positions, and then the participant had to match in a mirror image the position of the tested limb. Mean absolute distance error in the mediolateral and anteroposterior axis were obtained from Dexterit-E software, version 3.5.3. Absolute errors were compared with normative data (age and sex-matched) for each participant in the CRPS and HC groups.

Body perception: Body perception was assessed using the French version of the Bath Body Perception Disturbance Scale (Lewis and McCabe, 2010). Higher scores indicate higher body perception disturbances.

\section{Statistical Analysis}

Mean \pm standard deviation was reported in the results. Participants' characteristics (age, hand dominance, proprioception, sex) were compared between group (HC vs. CRPS) using independent t-tests and Fisher test for proportion.

For sensory and motor disturbances, non-parametric analyses of variance (nparLD) were used to assess Conflict sensitivity and motor disturbances. NparLD analyses are particularly relevant for small sample sizes, non-equivalent sample sizes between two groups and do not require normality of the data (Noguchi et al., 2012). In this analysis, the probability to observe higher scores in one condition compared to another is analyzed (rather than comparing mean scores as in parametric analyses).

For Conflict sensitivity, a three-way nparLD analysis was performed: Side (Affected/Non-dominant vs. Unaffected/ Dominant) X Categories of sensory disturbances (Category 1 vs. Category 2) X Group (CRPS vs. HC). For Motor disturbances, a three-way nparLD was performed: Side (Affected/Nondominant) X VF (Congruent vs. Incongruent) X Group (HC vs. CRPS).

Non-parametric Kendall coefficients were used to test the correlation between clinical characteristics (pain intensity, pain duration, proprioception and body perception) and Conflict sensitivity.

Analyses were made with $\mathrm{R}$ (version 3.5.2) with the nparLD package and the significance threshold was set at $\alpha<0.05$.

\section{RESULTS}

\section{Participants}

Sex (CRPS: 90\% women; HC: 91\% women, $p=0.99$ ) and selfreported hand dominance (CRPS: 90\% right-handed; HC: $86 \%$ right-handed, $p=0.79$ ) were equivalent between the two Groups. Age tended to be higher in the CRPS group than in the HC group, although this difference was not statistically significant (CRPS: $51 \pm 11$; HC: $43 \pm 11, p=0.08$ ). For proprioception, abnormal score relative to normative data was recorded in $30 \%$ of the CRPS participants while no HC had an abnormal score $(p<0.05)$. Table 1 presents clinical characteristics for the CRPS group.

\section{Objective 1: Sensory Disturbances}

The objective was to test whether Conflict sensitivity was higher and differed according to the Category of sensory disturbances in the Affected limb of individuals with CRPS compared to the 
TABLE 1 | Clinical characteristics.

\begin{tabular}{|c|c|c|c|c|c|c|c|c|c|c|c|c|c|}
\hline & Age & Sex & Laterality & $\begin{array}{l}\text { Affected } \\
\text { side }\end{array}$ & $\begin{array}{l}\text { Current } \\
\text { pain }\end{array}$ & $\begin{array}{l}\text { Pain } \\
24 h\end{array}$ & $\begin{array}{l}\text { Pain } \\
\text { week }\end{array}$ & Proprioception & BPDS & $\begin{array}{c}\text { Pain } \\
\text { onset } \\
\text { (months) }\end{array}$ & $\begin{array}{l}\text { Pharmacological } \\
\text { treatments }\end{array}$ & $\begin{array}{c}\text { Non- } \\
\text { pharmacological } \\
\text { treatments }\end{array}$ & $\begin{array}{c}\text { Actual } \\
\text { comorbidities }\end{array}$ \\
\hline CRPS01 & 60 & $\mathrm{~F}$ & Left & Right & 2 & 9 & 4 & Normal & 35 & 17 & $\begin{array}{l}\text { Gabapentin } \\
\text { pregabalin } \\
\text { Narcotic }\end{array}$ & & \\
\hline CRPS02 & 49 & $\mathrm{~F}$ & Right & Left & 4 & 6 & 5 & Normal & 15 & 18 & $\begin{array}{c}\text { Lidocaine } \\
\text { Pregabalin } \\
\text { Methotrimeprazine } \\
\text { Tapentadol }\end{array}$ & $\mathrm{PT}+\mathrm{OT}$ & \\
\hline CRPSO3 & 53 & $\mathrm{~F}$ & Right & Left & 3 & 3 & 4 & Normal & 12 & & $\begin{array}{l}\text { Morphin } \\
\text { Pregabalin }\end{array}$ & OT & \\
\hline CRPS04 & 50 & $\mathrm{~F}$ & Right & Left & 2 & 4 & 5 & Abnormal & 29 & 3 & $\begin{array}{l}\text { Pregabalin } \\
\text { Lidocaine }\end{array}$ & OT & \\
\hline CRPS05 & 39 & $\mathrm{H}$ & Right & Left & 2 & 5 & 4 & Normal & 11 & 5 & Naproxen & $\mathrm{PT}+\mathrm{OT}$ & \\
\hline CRPS06 & 59 & $\mathrm{~F}$ & Right & Left & 6 & 7 & 7 & Normal & 20 & 156 & $\begin{array}{l}\text { Pregabalin } \\
\text { Duloxetin }\end{array}$ & & Fibromyalgia \\
\hline CRPS07 & 54 & $\mathrm{~F}$ & Right & Left & 3 & 2 & 6 & Abnormal & 14 & 5 & Pregabalin & $\mathrm{PT}+\mathrm{OT}$ & $\begin{array}{l}\text { Adhesive } \\
\text { capsulitis }\end{array}$ \\
\hline CRPS08 & 25 & $\mathrm{~F}$ & Right & Right & 4 & 4 & 5 & Normal & 26 & 10 & $\begin{array}{l}\text { Pregabalin } \\
\text { Duloxetin } \\
\text { Morphine }\end{array}$ & $\mathrm{PT}+\mathrm{OT}$ & \\
\hline CRPS09 & 59 & $\mathrm{~F}$ & Right & Right & 1 & 5 & 5 & Abnormal & 27 & 4 & Pregabalin & OT & \\
\hline CRPS10 & 62 & $\mathrm{~F}$ & Right & Left & 0 & 3 & 3 & Normal & 17 & 4 & $\begin{array}{l}\text { Pregabalin } \\
\text { Paracetamol }\end{array}$ & OT & \\
\hline
\end{tabular}

M, male; F, female; PT, physiotherapy; O, occupational therapy; BPDS, body perception disturbance questionnaire; Laterality: self-reported hand dominance. 
TABLE 2 | Mean (standard deviation) for Conflict Sensitivity for each item of the sensory disturbance questionnaire.

\begin{tabular}{lccccc}
\hline & \multicolumn{2}{c}{ CRPS } & & \multicolumn{2}{c}{ HC } \\
\cline { 2 - 3 } \cline { 5 - 6 } & Affected & Unaffected & & Non-dominant & Dominant \\
\hline Category 1 & & & & & \\
$\quad$ Pain & $0.62(0.49)$ & $0(0)$ & & $0(0)$ & $0(0)$ \\
$\quad$ Discomfort & $0.70(0.79)$ & $0.47(0.95)$ & & $0.02(0.10)$ & $0.02(0.11)$ \\
Lost limb & $0.72(0.50)$ & $0.29(0.51)$ & & $0.17(0.33)$ & $0.26(0.56)$ \\
$\quad$ Weight & $0.35(0.34)$ & $0.25(0.30)$ & & $0(0.35)$ & $0.02(0.40)$ \\
$\quad$ Temperature & $0.36(0.59)$ & $0.26(0.24)$ & & $0(0.16)$ & $0.05(0.15)$ \\
Category 2 & & & & & \\
$\quad$ Peculiar & $0.10(0.24)$ & $0.55(0.47)$ & & $0.58(0.61)$ & $0.61(0.52)$ \\
$\quad$ Extra limb & $0.03(0.50)$ & $0.38(0.84)$ & & $0.31(0.49)$ & $0.40(0.77)$ \\
Losing control & $0.06(0.18)$ & $0.64(0.32)$ & & $0.54(0.78)$ & $0.55(0.67)$
\end{tabular}

CRPS, complex regional pain syndrome; HC, healthy controls.

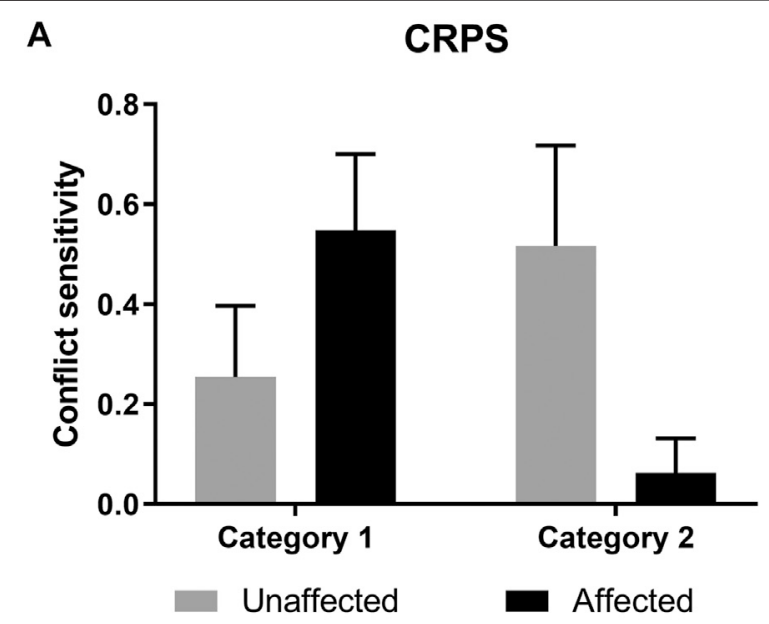

B

Healthy Controls

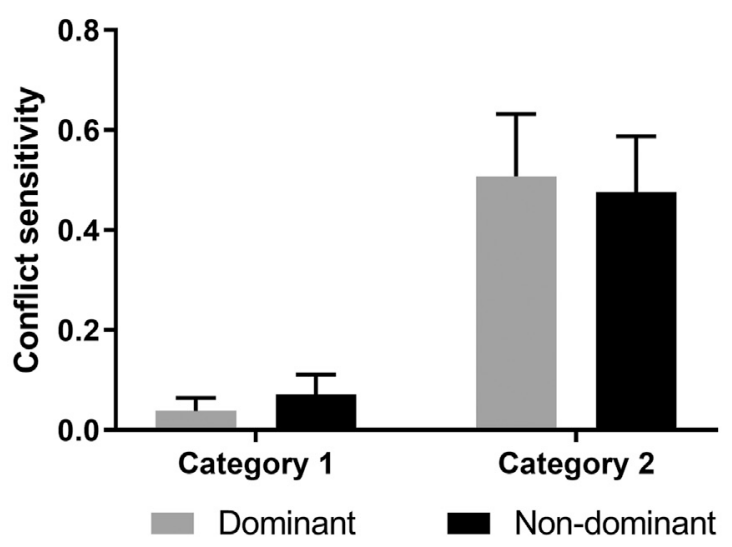

FIGURE 2 | Conflict sensitivity according to the CRPS (A) and Healthy Controls (B) groups. Category 1 = pain, discomfort, lost limb, temperature, weight; Category 2 = peculiarity, losing control, extra limb. Mean and standard deviation of the mean are reported.
TABLE $3 \mid p$-values of the nparLD analysis for Conflict sensitivity. Bold indicates significant effects. Italic indicates $p$-values for post-hoc analyses.

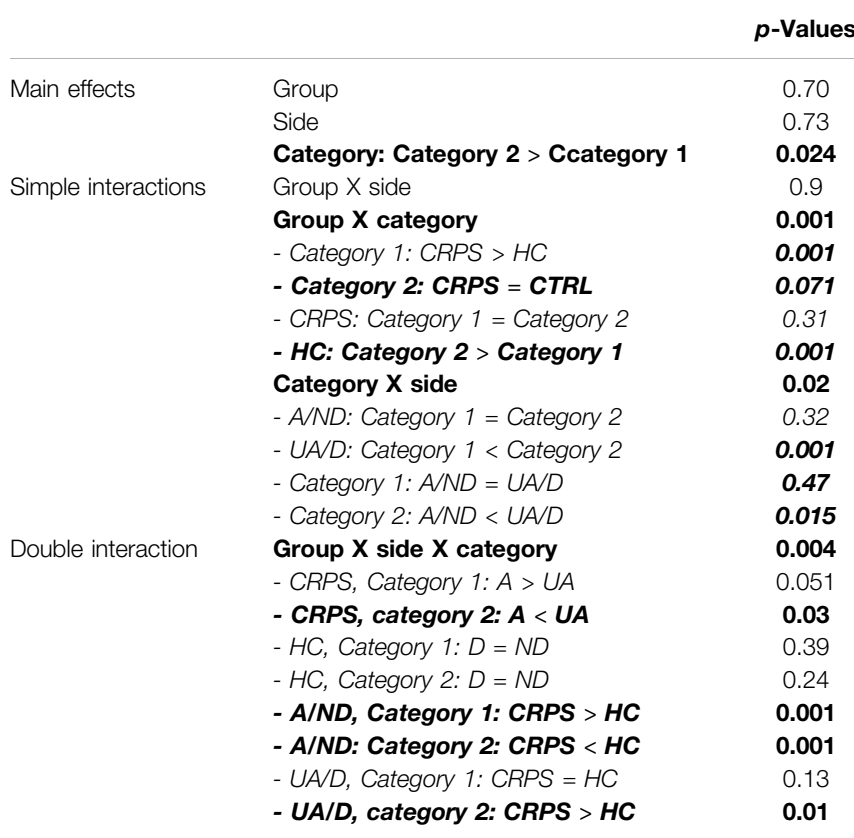

CRPS, complex regional pain syndrome; HC, healthy controls; $A$, affected; ND, nondominant; UA, unaffected; $D$, dominant.

Unaffected limb and HC. Table 2 describes the mean and standard deviation of Conflict sensitivity for each item of the sensory disturbance questionnaire.

Figure 2 shows Conflict sensitivity according to the Group, the Side and the Category of sensory disturbances. As our hypothesis focussed only on the double interaction (Side X Category $\mathrm{X}$ Group), only statistics for the double interaction are reported in the text. However, all statistics (main and interaction effects) are summarized in Table 3. The double interaction (Side X Category X Group) was significant ( $p=0.004)$ meaning that the pattern of Conflict sensitivity differed according to the Side, the Category of disturbances and the Group.

As expected, HC participants (Figure 2, lower panel) reported higher Category 2 than Category 1 disturbances for both limbs (both $p<0.001$ ). There was no significant difference between the Dominant and the Non-dominant limb for either Category 1 ( $p=$ $0.39)$ or Category $2(p=0.24)$ disturbances.

Contrary to HC, the intensity of Conflict sensitivity in CRPS (Figure 2A) differed according to the Category of disturbances and the Side in accordance with our hypothesis. Indeed, CRPS participants tended to report higher Category 1 disturbances for the Affected limb compared to the Unaffected limb $(p=0.051)$. The opposite pattern was observed for Category 2 disturbances, i.e., lower Conflict sensitivity was observed for the Affected limb compared to the Unaffected limb $(p<0.05)$.

The comparison between the Affected limb in CRPS and the Non-dominant limb in HC revealed a similar pattern as the comparison between both limbs in CRPS participants, since CRPS participants reported higher Category 1 disturbances for 
TABLE 4 | Correlation analyses. Bold indicates significant correlation.

\begin{tabular}{|c|c|c|c|c|}
\hline & Current pain & Pain last week & Body perception & $\begin{array}{c}\text { Time } \\
\text { since pain onset }\end{array}$ \\
\hline \multirow[t]{2}{*}{ Category 1} & $\tau=0.31$ & $\tau=0.56$ & $\tau=0$ & $\tau=0.30$ \\
\hline & $p=0.22$ & $p=0.03$ & $p=1$ & $p=0.54$ \\
\hline \multirow[t]{2}{*}{ Category 2} & $\tau=0.41$ & $\tau=0.33$ & $\tau=0.18$ & $\tau=0.14$ \\
\hline & $p=0.11$ & $p=0.21$ & $p=0.46$ & $p=0.24$ \\
\hline
\end{tabular}

the Affected limb compared to the Non-dominant limb in HC $(p<0.001)$ and lower Category 2 disturbances (Affected/CRPS $<$ Non-dominant/HC, $p<0.001)$.

Finally, we expected similar Conflict sensitivity between the Unaffected limb in CRPS and the Dominant limb in HC, for both Category 1 and Category 2 disturbances. No difference between CRPS and HC was observed for Category 2 disturbances (Unaffected/CRPS $=$ Dominant/HC, $p=0.13$ ). However, Category 1 disturbances were higher for the CRPS participants compared to HC (Unaffected/CRPS > Dominant/HC $p<0.01$ ).

\section{Objective 2: Correlation Between Clinical Characteristics and Conflict Sensitivity}

Results showed that higher Conflict sensitivity for Category 1 disturbances was related to higher pain intensity during the last week $(\tau=0.56, p=0.03)$. No other correlation was found to be significant (all $p>0.11$, Table 4).

\section{Objective 3: Motor Disturbances}

Incongruent VF significantly increased Drift $(p<0.001)$ and lowered the movement Amplitude $(p=0.004)$ compared to Congruent VF in all participants. Only for the Drift, a significant main effect of Group was observed $(p=0.041)$ indicating that CRPS had higher Drift than HC, but it was not specific for a limb or for a VF condition. Indeed, nor a significant effect of Side or interaction was observed (all $p>0.10$ ) for the Drift. For Amplitude there was no effect of Group, Side, or interaction (all $p>0.51$ ).

\section{DISCUSSION}

The origin of sensory disturbances in CRPS still remains unclear and it has been suggested that attentional and/or sensorimotor integration deficits are involved. In this study, we assessed whether sensory disturbances evoked by a unilateral sensorimotor conflict are specific to the Affected limb and differ according to the Category of sensory disturbances in individuals with a unilateral CRPS and HC. Our results demonstrate that CRPS participants report higher Category 1 than Category 2 disturbances for the Affected limb (while the reverse was observed for $\mathrm{HC}$ and for the Unaffected side). In addition, no difference was observed between the Unaffected limb in CRPS and the Dominant limb in HC for Category 2 disturbances, while higher conflict sensitivity was observed for Category 1 disturbances. Conflict sensitivity was related to higher pain (but not to other clinical characteristics) only for Category 1 disturbances. This pattern of results is better aligned with the sensorimotor integration hypothesis than with the hypothesis of a hypervigilance toward sensory disturbances since conflict sensitivity in CRPS is specific to Category 1 disturbances and to the Affected limb. However, the lack of intra- or inter-group difference in the motor disturbances evoked by the conflict somehow challenges the idea of sensorimotor integration deficits.

The uncertainty of results for deficits in sensorimotor integration in CRPS is well summarized in Bultitude and Petrini's study (Bultitude and Petrini, 2021). As noted by the authors, while alteration in body perception (Förderreuther et al., 2004; Lewis et al., 2007; Lewis and Schweinhardt, 2012) and motor performance (Maihöfner et al., 2003; Bank et al., 2014) are frequently observed in this population, some studies have failed to demonstrate deficits in sensorimotor integration (Turton et al., 2007; Reinersmann et al., 2013; Wang et al., 2019). However, these studies did not require active movements from the participants, which is a strong limitation to study sensorimotor integration. A very few studies have investigated sensorimotor integration during active movements in CRPS and focus either on sensory perception (Brun et al., 2018b; Brun et al., 2018c), motor performance (Verfaille et al., 2020; Bultitude and Petrini, 2021) or both (Tajadura-Jiménez et al., 2017). Results of these studies have consistently shown alterations in perception, while alterations in motor performance are inconsistent across studies. For sensory perception, our results replicate a previous study showing that individuals with CRPS and fibromyalgia are more sensitive to sensorimotor conflicts, specifically for Category 1 sensory disturbances (i.e., items related to an increase of pain, discomfort, a change in weight and temperature, and the feeling of losing a limb) compared to pain-free individuals and people with arthritis (Brun et al., 2018c). Moreover, the sense of limb movement during active movements is altered in CRPS and is characterized by blurred representation of the movement's limb (Brun et al., 2018b). Another study investigated integration of auditory stimuli during walking and demonstrated that real-time alterations of the sound produced by walking increase body perception disturbances and pain in CRPS (Tajadura-Jiménez et al., 2017), and this effect seems to be larger and more variable than the previously described effect in healthy individuals (Tajadura-Jiménez et al., 2015). In addition to these sensory perturbations, altered gait biomechanics (stance and swing) was observed during these alterations of auditory feedback, but this effect appeared to be similar for CRPS and healthy individuals (Tajadura-Jiménez et al., 2015; TajaduraJiménez et al., 2017). Two other studies investigated sensorimotor integration during active movements with 
measures of motor performance in CRPS participants compared to controls (Verfaille et al., 2020; Bultitude and Petrini, 2021). Verfaille and collaborators demonstrated that CRPS participants make more errors than controls during a reaching task that requires the participant to integrate visual and proprioceptive feedback. Interestingly, this increase in errors was not explained by isolated deficits in visual or proprioceptive processing, but rather by a difficulty to integrate both sources of information during movement (Verfaille et al., 2020). However, Bultitude and Petrini found that people with CRPS can optimally integrate visual and self-motion cues under some conditions, but use different strategies to controls (Bultitude and Petrini, 2021). Overall, it appears that sensory perturbations arising from sensorimotor integration problems in CRPS do not necessarily result in deficits in motor performance, a result that was also found in fibromyalgia (Brun et al., 2020) and in healthy individuals with experimental pain (Brun et al., 2017), potentially due to adaptations in motor strategies employed.

While our results suggest deficits in sensorimotor integration, it is not possible to totally exclude the attentional hypothesis. Attentional bias in chronic pain might be expressed in hypervigilance toward the pain and pain-related information (Hollins et al., 2009), and/or a bias away from the painful limb (Broadbent et al., 2021). Spatial neglect has been well studied in CRPS (for a systematic review and meta-analysis see (Broadbent et al., 2021)), but hypervigilance for pain and other pain-related information in CRPS was poorly investigated (Broadbent et al., 2021). In our study, higher Category 1 sensations for the Unaffected limb were observed in CRPS compared to HC, which suggests hypervigilance toward Category 1 disturbances in CRPS. However, the fact that higher Category 1 disturbances are observed for the Affected limb compared to the Unaffected limb in CRPS suggest that hypervigilance cannot completely explain sensory disturbances in CRPS.

Some limitations need to be highlighted. A convenience sample was used and a small number of participants was recruited in the CRPS group, yielding the possibility of type II error. Note however that nparLD analyses are well designed for small sample sizes. Difficulty in recruitment was explained by the choice of the experimental set-up (KINARM exoskeleton system). First, this system requires the participants to be positioned with $80^{\circ}$ of shoulder abduction while performing forward movements (in the horizontal plane) of $30 \mathrm{~cm}$ (the UL being fully supported by the exoskeleton). This limits the inclusion of participants with shoulder deficits. Second, participants with a body mass index superior to 30 can typically not be appropriately fitted in the KINARM, also leading to the exclusion of some participants. These limitations both tend to bias the sample toward individuals with milder CRPS. Despite this, significant differences were observed for sensory disturbances. However, this might contribute to the absence of significant results for motor disturbances. In addition, the effects of treatments (pharmacological and nonpharmacological) have not been controlled and might interfere with the results. Finally, the affected limb of CRPS participants was compared to the nondominant limb of the controls, irrespective of the hand dominance of the CRPS participants. This approach, although commonly used in the literature, presents some limitations. However, given the absence of significant difference between both UL and HC, it appears unlikely that this approach has influenced the results.

In conclusion, a unilateral sensorimotor conflict induces higher Category 1 sensory disturbances in the Affected limb compared to both the Unaffected limb in CRPS and the Non-Dominant limb in HC. Therefore, conflict-induced sensory disturbances are specific in terms of the Category of sensation evoked and in terms of localization, suggesting that sensory disturbances in CRPS depend on sensorimotor integration deficits rather than hypervigilance toward pain and pain-related information. However, motor disturbances were similar between CRPS and HC, which suggests that the motor response to the sensorimotor conflict is not impaired. Future work should evaluate the effect of interventions on sensory disturbances in CRPS, to determine whether they disappear in patients who recover.

\section{DATA AVAILABILITY STATEMENT}

The raw data supporting the conclusion of this article will be made available by the authors, without undue reservation.

\section{ETHICS STATEMENT}

The studies involving human participants were reviewed and approved by the Centre intégré universitaire en santé et services sociaux de la Capitale Nationale. The patients/participants provided their written informed consent to participate in this study.

\section{AUTHOR CONTRIBUTIONS}

$\mathrm{CB}, \mathrm{CSM}$, and $\mathrm{CM}$ designed the study; $\mathrm{CB}$ and $\mathrm{AP}$ performed data collection; $\mathrm{CB}, \mathrm{CSM}$, and CM analyzed and interpreted the data; $\mathrm{CB}$ and $\mathrm{CM}$ drafted the paper; $\mathrm{CB}, \mathrm{AP}, \mathrm{CSM}$, and $\mathrm{CM}$ commented on the paper and approved the final version.

\section{FUNDING}

CB was supported by fellowships from the Centre interdisciplinaire de recherche en réadaptation et en intégration sociale (CIRRIS), from the Centre thématique de recherche en neurosciences (CTRN), from the Faculté de médecine de l'Université Laval and from Fonds de recherche Québec-Nature et Technologies (FRQNT). CM is supported by an Emeritus Research Scholar Award from Fonds de recherche Québec-Santé (FRQS) (grant number 251649).

\section{ACKNOWLEDGMENTS}

The authors thank Nicolas Robitaille for the development and implementation of the task and Martin Gagné, for the development of the Matlab scripts. 


\section{REFERENCES}

Bank, P. J. M., Peper, C., Lieke, E., Marinus, J., Beek, P. J., and van Hilten, J. J. (2013). Motor Dysfunction of Complex Regional Pain Syndrome Is Related to Impaired Central Processing of Proprioceptive Information. J. Pain 14, 1460-1474. doi:10.1016/j.jpain.2013.07.009

Bank, P. J. M., van Rooijen, D. E., Marinus, J., Reilmann, R., and van Hilten, J. J. (2014). Force Modulation Deficits in Complex Regional Pain Syndrome: a Potential Role for Impaired Sense of Force Production. Eur. J. Pain 18, 1013-1023. doi:10.1002/j.1532-2149.2013.00446.x

Bean, D. J., Johnson, M. H., Heiss-Dunlop, W., Lee, A. C., and Kydd, R. R. (2015). Do psychological Factors Influence Recovery from Complex Regional Pain Syndrome Type 1? A Prospective Study. Pain 156, 2310-2318. doi:10.1097/ j.pain.0000000000000282

Broadbent, P., Liossi, C., and Schoth, D. E. (2021). Attentional Bias to Somatosensory Stimuli in Chronic Pain Patients: a Systematic Review and Meta-Analysis. Pain 162, 332-352. doi:10.1097/ j.pain.0000000000002040

Brun, C., Gagné, M., McCabe, C. S., and Mercier, C. (2018a). Motor and Sensory Disturbances Induced by Sensorimotor Conflicts during Passive and Active Movements in Healthy Participants. PLoS One 13, e0203206-15. doi:10.1371/ journal.pone.0203206

Brun, C., Gagné, M., McCabe, C. S., and Mercier, C. (2017). Sensory Disturbances, but Not Motor Disturbances, Induced by Sensorimotor Conflicts Are Increased in the Presence of Acute Pain. Front. Integr. Neurosci. 11, 1-13. doi:10.3389/ fnint.2017.00014

Brun, C., Giorgi, N., Pinard, A.-M., Gagné, M., McCabe, C. S., and Mercier, C. (2018b). Exploring the Relationships between Altered Body Perception, Limb Position Sense, and Limb Movement Sense in Complex Regional Pain Syndrome. The J. Pain 20, 17-27. doi:10.1016/j.jpain.2018.07.008

Brun, C., McCabe, C. S., Mercier, C., Mccabe, S., and Mercier, C. (2020). The Contribution of Motor Commands to the Perturbations Induced by Sensorimotor Conflicts in Fibromyalgia. Neuroscience 434, 55-65. doi:10.1016/j.neuroscience.2020.03.017

Brun, C., Mercier, C., Grieve, S., Palmer, S., Bailey, J., and McCabe, C. S. (2018c). Sensory Disturbances Induced by Sensorimotor Conflicts Are Higher in Complex Regional Pain Syndrome and Fibromyalgia Compared to Arthritis and Healthy People, and Positively Relate to Pain Intensity. Eur. J. Pain 23, 483-494. doi:10.1002/ejp.1322

Bultitude, J. H., and Petrini, K. (2021). Altered Visuomotor Integration in Complex Regional Pain Syndrome. Behav. Brain Res. 397, 112922. doi:10.1016/ j.bbr.2020.112922

Cisler, J. M., and Koster, E. H. W. (2010). Mechanisms of Attentional Biases towards Threat in Anxiety Disorders: An Integrative Review. Clin. Psychol. Rev. 30, 203-216. doi:10.1016/j.cpr.2009.11.003

Di Pietro, F., McAuley, J. H., Parkitny, L., Lotze, M., Wand, B. M., Moseley, G. L., et al. (2013). Primary Somatosensory Cortex Function in Complex Regional Pain Syndrome: A Systematic Review and Meta-Analysis. J. Pain 14, 1001-1018. doi:10.1016/j.jpain.2013.04.001

Don, S., Voogt, L., Meeus, M., De Kooning, M., and Nijs, J. (2016). Sensorimotor Incongruence in People with Musculoskeletal Pain: A Systematic Review. Pain Pract. 17, 115-128. doi:10.1111/papr.12456

Drummond, P. D., Finch, P. M., Birklein, F., Stanton-Hicks, M., and Knudsen, L. F. (2001). Hemisensory Disturbances in Patients With Complex Regional Pain Syndrome. Pain 159, 1824-1832. doi:10.1097/j.pain.0000000000001280

Dukelow, S. P., Herter, T. M., Moore, K. D., Demers, M. J., Glasgow, J. I., and Bagg, S. D. (2001). Quantitative Assessment of Limb Position Sense Following Stroke. J. Neurorehabil Neural Repair 24 (2), 178-187. doi:10.1177/1545968309345267

Förderreuther, S., Sailer, U., and Straube, a. (2004). Impaired Self-Perception of the Hand in Complex Regional Pain Syndrome (CRPS). Pain 110, 756-761. doi:10.1016/j.pain.2004.05.019

Frettlöh, J., Hüppe, M., and Maier, C. (2006). Severity and Specificity of Neglectlike Symptoms in Patients with Complex Regional Pain Syndrome (CRPS) Compared to Chronic Limb Pain of Other Origins. Pain 124, 184-189. doi:10.1016/j.pain.2006.04.010

Harden, N. R., Bruehl, S., Perez, R. S. G. M., Birklein, F, Marinus, J., Maihofner, C., et al. (2010). Validation of Proposed Diagnostic Criteria (The "Budapest
Criteria") for Complex Regional Pain Syndrome. Pain 150, 268-274. doi:10.1016/j.pain.2010.04.030

Harden, R. N., Bruehl, S., Stanton-Hicks, M., and Wilson, P. R. (2007). Proposed New Diagnostic Criteria for Complex Regional Pain Syndrome. Pain Med. 8, 326-331. doi:10.1111/j.1526-4637.2006.00169.x

Harris, A. J. (1999). Cortical Origin of Pathological Pain. The Lancet 354, 1464-1466. doi:10.1016/s0140-6736(99)05003-5

Hollins, M., Harper, D., Gallagher, S., Owings, E. W., Lim, P. F., Miller, V., et al. (2009). Perceived Intensity and Unpleasantness of Cutaneous and Auditory Stimuli: An Evaluation of the Generalized Hypervigilance Hypothesis. Pain 141, 215-221. doi:10.1016/j.pain.2008.10.003

Knudsen, L., Finch, P. M., Drummond, P. D., et al. (2011). The Specificity and Mechanisms of Hemilateral Sensory Disturbances in Complex Regional Pain Syndrome. J. Pain 12, 985-990. doi:10.1016/j.jpain.2011.03.001

Kuczynski, A., Dukelow, S., Yajure, J., Roe, J., and Kirton, A. (2014). Robotic Quantification of Proprioceptive Dysfunction in Children with Perinatal Stroke. Stroke 45, E261.

Lewis, J. S., Kersten, P., McCabe, C. S., McPherson, K. M., and Blake, D. R. (2007). Body Perception Disturbance: A Contribution to Pain in Complex Regional Pain Syndrome (CRPS). Pain 133, 111-119. doi:10.1016/ j.pain.2007.03.013

Lewis, J. S., and McCabe, C. S. (2010). Correcting the Body in Mind: Body Perception Disturbance in Complex Regional Pain Syndrome (CRPS) and Rehabilitation Approaches. Pract. Pain Manag. 10, 60-66.

Lewis, J. S., and Schweinhardt, P. (2012). Perceptions of the Painful Body: The Relationship between Body Perception Disturbance, Pain and Tactile Discrimination in Complex Regional Pain Syndrome. Ejp 16, 1320-1330. doi:10.1002/j.1532-2149.2012.00120.x

Maihöfner, C., Handwerker, H. O., Neundörfer, B., and Birklein, F. (2003). Patterns of Cortical Reorganization in Complex Regional Pain Syndrome. Neurology 61, 1707-1715. doi:10.1212/01.wnl.0000098939.02752.8e

McCabe, C. S., and Blake, D. R. (2008). An Embarrassment of Pain Perceptions? towards an Understanding of and Explanation for the Clinical Presentation of CRPS Type 1. Rheumatology 47, 1612-1616. doi:10.1093/rheumatology/ ken 254

McCabe, C. S., Cohen, H., and Blake, D. R. (2007). Somaesthetic Disturbances in Fibromyalgia Are Exaggerated by Sensory Motor Conflict: Implications for Chronicity of the Disease? Rheumatology 46, 1587-1592. doi:10.1093/ rheumatology/kem204

McCabe, C. S., Cohen, H., Hall, J., Lewis, J., Rodham, K., and Harris, N. (2009). Somatosensory Conflicts in Complex Regional Pain Syndrome Type 1 and Fibromyalgia Syndrome. Curr. Rheumatol. Rep. 11, 461-465. doi:10.1007/ s11926-009-0067-4

McDermid, A. J., Rollman, G. B., and McCain, G. A. (1996). Generalized Hypervigilance in Fibromyalgia: Evidence of Perceptual Amplification. Pain 66, 133-144. ST-Generalized hypervigilance in fybrom. doi:10.1016/03043959(96)03059-x

Michal, M., Adler, J., Reiner, I., Wermke, A., Ackermann, T., Schlereth, T., et al. (2017). Association of Neglect-like Symptoms with Anxiety, Somatization, and Depersonalization in Complex Regional Pain Syndrome. Pain Med. Pnw 18 (4), 764-772. doi:10.1093/pm/pnw214

Noguchi, K., Gel, Y. R., Brunner, E., and Konietschke, F. (2012). nparLD: An $\mathrm{R}$ Software Package for the Nonparametric Analysis of Longitudinal Data in Factorial Experiments. J. Stat. Softw. 50 (12), 1-23. doi:10.18637/ jss.v050.i12

Palmer, S., Bailey, J., Brown, C., Jones, A., and McCabe, C. S. (2019). Sensory Function and Pain Experience in Arthritis, Complex Regional Pain Syndrome, Fibromyalgia Syndrome, and Pain-Free Volunteers: A Cross-Sectional Study. Clin. J. Pain 35, 894-900. doi:10.1097/AJP.0000000000000751

Reinersmann, A., Landwehrt, J., Krumova, E. K., Peterburs, J., Ocklenburg, S. Güntürkün, O., et al. (2013). The Rubber Hand Illusion in Complex Regional Pain Syndrome: Preserved Ability to Integrate a Rubber Hand Indicates Intact Multisensory Integration is. Pain 154, 1519-1527. doi:10.1016/ j.pain.2013.03.039

Rommel, O., Malin, J. P., Zenz, M., and Jänig, W. (2001). Quantitative Sensory Testing, Neurophysiological and Psychological Examination in Patients With Complex Regional Pain Syndrome and Hemisensory Deficits. Pain 93, 279-293. doi:10.1016/s0304-3959(98)00202-4 
Scott, S., and Brown, I. (2013). Method and Apparatus for Assessing Proprioceptive Function. US Pat 20.

Tajadura-Jiménez, A., Basia, M., Deroy, O., Fairhurst, M., Marquardt, N., and Bianchi-Berthouze, N. (2015). "As Light as Your Footsteps: Altering Walking Sounds to Change Perceived Body Weight, Emotional State and Gait". In Conf Hum Factors Comput Syst - Proc, 2943-2952. doi:10.1145/ 2702123.2702374

Tajadura-Jiménez, A., Cohen, H., and Bianchi-Berthouze, N. (2017). Bodily Sensory Inputs and Anomalous Bodily Experiences in Complex Regional Pain Syndrome: Evaluation of the Potential Effects of Sound Feedback. Front. Hum. Neurosci. 11, 379. doi:10.3389/fnhum.2017.00379

Turton, A. J., McCabe, C. S., Harris, N., and Filipovic, S. R. (2007). Sensorimotor Integration in Complex Regional Pain Syndrome: A Transcranial Magnetic Stimulation Study. Pain 127, 270-275. doi:10.1016/j.pain.2006.08.021

Vartiainen, N., Kirveskari, E., Kallio-Laine, K., Kalso, E., and Forss, N. (2009). Cortical Reorganization in Primary Somatosensory Cortex in Patients with Unilateral Chronic Pain. J. Pain 10, 854-859. doi:10.1016/ j.jpain.2009.02.006
Verfaille, C., Filbrich, L., Rossetti, Y., Berquin, A., Mouraux, D., and Barbier, O. (2020). Visuomotor Impairments in Complex Regional Pain Syndrome during Pointing Tasks. Pain 162 (3), 811-822. doi:10.1097/j.pain.0000000000002068

Wang, A. P., Butler, A. A., Valentine, J. D., Rae, C. D., Mcauley, J. H., Gandevia, S. C., et al. (2019). A Novel Finger Illusion Reveals Reduced Weighting of Bimanual Hand Cortical Representations in People with Complex Regional Pain Syndrome. J. Pain 20, 171-180. doi:10.1016/ j.jpain.2018.08.008

Conflict of Interest: The authors declare that the research was conducted in the absence of any commercial or financial relationships that could be construed as a potential conflict of interest.

Copyright $\odot 2021$ Brun, Pinard, McCabe and Mercier. This is an open-access article distributed under the terms of the Creative Commons Attribution License (CC BY). The use, distribution or reproduction in other forums is permitted, provided the original author(s) and the copyright owner(s) are credited and that the original publication in this journal is cited, in accordance with accepted academic practice. No use, distribution or reproduction is permitted which does not comply with these terms. 